\title{
Identification of the Point Sources in Some Stochastic Wave Equations
}

\author{
Yaozhong $\mathrm{Hu}^{1}$ and Guanglin Rang ${ }^{2}$ \\ ${ }^{1}$ Department of Mathematics, University of Kansas, Lawrence, KS 66045, USA \\ ${ }^{2}$ School of Mathematics and Statistics, Wuhan University, Wuhan 430072, China \\ Correspondence should be addressed to Guanglin Rang; glrang.math@whu.edu.cn
}

Received 24 September 2013; Accepted 21 March 2014; Published 22 April 2014

Academic Editor: Yiming Ding

Copyright (c) 2014 Y. Hu and G. Rang. This is an open access article distributed under the Creative Commons Attribution License, which permits unrestricted use, distribution, and reproduction in any medium, provided the original work is properly cited.

\begin{abstract}
We introduce and study a type of (one-dimensional) wave equations with noisy point sources. We first study the existence and uniqueness problem of the equations. Then, we assume that the locations of point sources are unknown but we can observe the solution at some other location continuously in time. We propose an estimator to identify the point source locations and prove the convergence of our estimator.
\end{abstract}

\section{Introduction}

Assume that there are a certain number of objects in a certain area of ocean or other media. The total number of the objects and the location of each object are unknown. We need to identify the total number and the precise locations of the objects. The objects are also assumed to emit (point source) sound waves and we are able to measure these sound waves received in some known locations. The objective is to use these measurements for our identification problem.

This type of problem has been studied mathematically in the framework of inverse problems for partial differential equations (wave equations). The sound travels according to the following second-order wave equation with point sources:

$$
\begin{gathered}
\frac{\partial^{2} u(t, x)}{\partial t^{2}}-\sum_{i=1}^{d} \frac{\partial^{2} u(t, x)}{\partial x_{i}^{2}}=\sum_{k=1}^{N} \sigma_{k} \delta\left(x-y_{k}\right) \\
u(0, x)=0, \quad \frac{\partial u}{\partial t}(0, x)=0,
\end{gathered}
$$

where $x \in \mathbb{R}^{d}, t \in(0, T), N \in \mathbb{N}$, and $y_{1}, \ldots, y_{N}$ are some given points in $\mathbb{R}^{d}, \delta(x)$ is the Dirac delta function, and $\sigma_{k}$, $k=1,2, \ldots, N$, are some known constants. The solution $(u(t, x), \quad x \in D, 0 \leq t \leq T)$ is supposed to be known for some space points $D$ and for some interval $[0, T]$. The total number $N$ and each location $y_{k}$ of point sources are estimated from $(u(t, x), x \in D, 0 \leq t \leq T)$. We refer to, for example, [1] and in particular the references therein for some recent study in this area. This theory has found substantial applications in determining the heat sources in heat conduction, the magnetic sources in brain, the earthquake sources of seismic waves, and so on.

In practice, the sound wave travels inevitably under some influence of noises. Voluntarily or involuntarily, the point sources themselves may also emit noises to avoid being detected. Thus, we are led to the following stochastic wave equations:

$$
\begin{aligned}
& \frac{\partial^{2} u(t, x)}{\partial t^{2}}-\sum_{i=1}^{d} \frac{\partial^{2} u(t, x)}{\partial x_{i}^{2}} \\
& =b(t, x, u(t, x))+\sigma_{0}(t, x, u) \dot{B}_{0}(t, x) \\
& \quad+\sum_{k=1}^{N} \sigma_{k}(t, x, u) \delta\left(x-y_{k}\right) \dot{B}_{k}(t, x) \\
& u(0, x)=v_{0}(x), \quad \frac{\partial u}{\partial t}(0, x)=\bar{v}_{0}(x),
\end{aligned}
$$

where $x \in \mathbb{R}^{d}, t \in(0, T), N \in \mathbb{N}$, and $y_{1}, \ldots, y_{N}$ are some given points in $\mathbb{R}^{d}, \delta(x)$ is the Dirac delta function, $v_{0}$ and $v_{1}(x)$ are two given deterministic functions, and $\dot{B}_{k}$, 
$k=0,1,2, \ldots, N$, are independent Gaussian noises which are white in time and correlated in space.

When $\sigma_{1}=\cdots=\sigma_{k}=0$, the stochastic wave equation (2) has been studied since long time. Let us mention the first lecture note [2] and a recent lecture note [3]. Many properties such as the sample path Hölder continuity of the solution are obtained (see $[4,5]$ and the references therein).

However, when $\sigma_{i}, i=1, \ldots, N$, are not all zero, then (2) is highly singular because of the presence of the Dirac delta functions multiplied by the Gaussian noises. Such equation has not been studied yet. The first objective of this paper is to give the definition of the solution to such an equation and to show the existence of uniqueness of the solution under some appropriate conditions. This will be done in Section 2. Since the case when $\sigma_{1}=\cdots=\sigma_{N}=0$ has been well studied, we will now assume $\sigma_{0}=0$ to simplify our presentation. However, since our objective is the identification of the $N$ and point source positions $y_{1}, \ldots, y_{N}$, we will not go to spend too much effort here. For this reason, we restrict ourselves to only one space dimension case. We will present higher space dimension case in another project.

To well explain our approach of identification, we will further restrict our model. We will consider the special case of (2) where the coefficients $\sigma_{k}(t, x, u)=\rho_{k}(t)$ are independent of $x$ and $u$ and $v_{0}=\bar{v}_{0}=0$. Namely, we will concentrate on the stochastic wave equation of the form

$$
\begin{gathered}
\frac{\partial^{2} u(t, x)}{\partial t^{2}}-\sum_{i=1}^{d} \frac{\partial^{2} u(t, x)}{\partial x_{i}^{2}}=\sum_{k=1}^{N} \rho_{k}(t) \delta\left(x-y_{k}\right) \dot{B}_{k}(t, x) \\
u(0, x)=0, \quad \frac{\partial u}{\partial t}(0, x)=0 .
\end{gathered}
$$

In this case, we will write down the explicit expression of the solution. It will be done in Section 3. In this section, we also obtain some properties of the solution which will be useful in the later section of the paper.

Now, we assume that, in (3), the total number of point sources $N$ and the positions $y_{i}, i=1, \ldots, N$, are unknown. However, we are able to observe the sound signal received at some given known locations $x_{1}, \ldots, x_{m}$, continuously in the time interval $[0, T]$. Namely, we assume that $\left(u\left(t, x_{1}\right), \ldots, u\left(t, x_{m}\right), 0 \leq t \leq T\right)$ are known. We would like to use this information to identify $N$ and $y_{i}$, $i=1, \ldots, N$. In Section 4, we will develop a new approach to obtain some statistical estimators $\widehat{N}$ and $\widehat{y}_{1}, \widehat{y}_{2}, \ldots, \widehat{y}_{N}$ to estimate the total number $N$ and the locations $y_{1}, y_{2}, \ldots, y_{N}$ of the point sources. The approach combines the reciprocity gap functional approach from the theory of partial differential equations with theory from stochastic processes. We show the almost sure convergence of our estimators $\widehat{N}$ and $\widehat{y}_{1}, \widehat{y}_{2}, \ldots, \widehat{y}_{N}$ to the true parameters $N$ and $y_{1}, y_{2}, \ldots, y_{N}$.

\section{Stochastic Wave Equations with Noisy Point Sources}

Let $(\Omega, \mathscr{F}, P)$ be a basic probability space with right continuous filtration of $\sigma$-algebras $\left\{\mathscr{F}_{t}, t \geqslant 0\right\}$ satisfying the usual conditions. Let $B(t, x)=\left(B_{1}(t, x), B_{2}(t, x), \ldots, B_{N}(t, x)\right), t \geqslant$
$0, x \in \mathbb{R}^{d}$ be $N$ dimensional Gaussian random fields. The formal derivatives $\dot{B}_{k}(t, x):=\left(\partial^{d+1} / \partial t \partial x_{1} \cdots \partial x_{d}\right) B_{k}(t, x)$, $k=1,2, \ldots, N$, are called the Gaussian noises. We assume that these Gaussian noises are white in time and correlated in space with covariance $r(x, y)$ (see [5]). Namely, we assume that

$$
\mathbb{E}\left[\dot{B}_{k}(t, x) \dot{B}_{k}(s, y)\right]=\delta(t-s) r_{k}(x, y),
$$

where $\mathbb{E}$ denotes the expectation on $(\Omega, \mathscr{F}, P), r_{k}: \mathbb{R}^{d} \times \mathbb{R}^{d} \rightarrow$ $\mathbb{R}$, and $(k=1,2, \ldots, N)$ are some symmetric positive definite functions of $x$ and $y$. This is interpreted as

$$
\begin{aligned}
\mathbb{E} & {\left[B_{k}(t, x) B_{k}(s, y)\right] } \\
& =(t \wedge s) \int_{0}^{x_{1}} \cdots \int_{0}^{x_{d}} \int_{0}^{y_{1}} \cdots \int_{0}^{y_{d}} r_{k}(u, v) \mathrm{d} u \mathrm{~d} v .
\end{aligned}
$$

For any (deterministic) smooth function $\varphi$ in $\mathscr{C}_{0}^{\infty}([0, T] \times$ $\left.\mathbb{R}^{d}\right)$, the stochastic integral $B_{k}(\varphi)=\int_{0}^{T} \int_{\mathbb{R}^{d}} \varphi(s, x) B_{k}(\mathrm{~d} s, \mathrm{~d} x)$ is well-defined in the sense of Walsh ([2]). The following fact is well-known: for any two smooth functions $\varphi$ and $\psi$ in $\mathscr{C}_{0}^{\infty}\left([0, T] \times \mathbb{R}^{d}\right)$, we have

$$
\begin{aligned}
\mathbb{E} & \left(B_{k}(\varphi) B_{k}(\psi)\right) \\
= & \int_{0}^{T} \mathrm{~d} s \iint_{\mathbb{R}^{d}} \varphi(s, x) r_{k}(x, y) \psi(s, y) \mathrm{d} x \mathrm{~d} y .
\end{aligned}
$$

We also call $\mathbb{E}\left(B_{k}(\varphi) B_{k}(\psi)\right)$ the covariance functional of $\dot{B}_{k}$, denoted by $J_{k}(\varphi, \psi)$.

Sometimes, we also use the Fourier transform theory to study the stochastic integral and the stochastic equations. We use $\mathscr{F} \varphi(s, \cdot)(\xi)=\int_{\mathbb{R}^{d}} e^{2 \pi i x \xi} \varphi(s, x) \mathrm{d} x$ to denote the Fourier transformation of $\phi(s, \cdot)$ and $\widetilde{\varphi}(s, x)=\varphi(s,-x)$.

If we assume that the noise is spatially homogeneous, that is, $r_{k}(x, y)=r_{k}(x-y)$, then there exists a nonnegative tempered measure $\mu_{k}$ which is the Fourier transform of $r_{k}(x) \mathrm{d} x$. With this notation, we can also write

$$
J_{k}(\varphi, \psi)=\int_{0}^{\infty} \mathrm{d} s \int_{\mathbb{R}^{d}} \mu_{k}(\mathrm{~d} \xi) \mathscr{F} \varphi(s, \cdot)(\xi) \mathscr{F} \widetilde{\psi}(s, \cdot)(\xi) .
$$

From the general theory of stochastic integral (see, e.g., [3]), we see that if $\left(g(t, x), 0 \leq t \leq T, x \in \mathbb{R}^{d}\right)$ is a real valued $\mathscr{F}_{t}$-adapted process such that

$$
\int_{0}^{T} \mathrm{~d} s \iint_{\mathbb{R}^{d}} r_{k}(x, y) \mathbb{E}[|g(s, x)||g(s, y)|] \mathrm{d} x d y<\infty,
$$

then the stochastic integral $\int_{0}^{T} \int_{\mathbb{R}^{d}} g(t, x) B_{k}(d t, \mathrm{~d} x)$ is welldefined and

$$
\begin{aligned}
\mathbb{E}\left[\int_{0}^{T} \int_{\mathbb{R}^{d}} g(s, x) B_{k}(\mathrm{~d} s, \mathrm{~d} x)\right]^{2} \\
\quad=\int_{0}^{T} \mathrm{~d} s \iint_{\mathbb{R}^{d}} r_{k}(x, y) \mathbb{E}[g(s, x) g(s, y)] \mathrm{d} x d y .
\end{aligned}
$$

Now, let $y$ be a given fixed point in $\mathbb{R}^{d}$ and let $(\lambda(t, x), 0 \leq t \leq$ $T, x \in \mathbb{R}^{d}$ ) be a real valued $\mathscr{F}_{t}$-adapted process. We want to 
define the stochastic integral $\int_{0}^{t} \int_{\mathbb{R}^{d}} \lambda(s, x) \delta(x-y) F(\mathrm{~d} s \mathrm{~d} x)$, where $\delta(x-y)$ is the Dirac delta function. To this end, we will use smooth functions to approximate the Dirac delta function.

Let $\phi$ be a smooth function with compact support $K=$ $[-1,1]^{d}$. Set $\phi_{n}(x-y)=n^{d} \phi(n(x-y))$. It is clear that $\phi_{n}$ converges to $\delta(x-y)$ in the sense of distribution as $n \rightarrow \infty$. For each $n$, the stochastic integral

$$
I_{n}:=\int_{0}^{T} \int_{\mathbb{R}^{d}} \lambda(s, x) \phi_{n}(x-y) B_{k}(\mathrm{~d} s, \mathrm{~d} x)
$$

is well-defined. We want to know whether $I_{n}$ has a limit in $L^{2}(\Omega, \mathscr{F}, P)$ or not. First, we have the following computations:

$$
\begin{aligned}
& \mathbb{E}\left[I_{n}-I_{m}\right]^{2} \\
& =\mathbb{E}\left(\int _ { 0 } ^ { T } \int _ { \mathbb { R } ^ { d } } \lambda ( s , x ) \left[\phi_{n}(x-y)\right.\right. \\
& \left.\left.-\phi_{m}(x-y)\right] B_{k}(\mathrm{~d} s, \mathrm{~d} x)\right)^{2} \\
& =\int_{0}^{T} \iint_{\mathbb{R}^{d}} \mathbb{E}[\lambda(s, x) \lambda(s, z)] \\
& \quad \times\left(\phi_{n}(x-y)-\phi_{m}(x-y)\right) \\
& \quad \times\left(\phi_{n}(z-y)-\phi_{m}(z-y)\right) \\
& \quad \times r_{k}(x, z) \mathrm{d} x \mathrm{~d} z \mathrm{~d} s .
\end{aligned}
$$

Passing the difference $\phi_{n}(x-y)-\phi_{m}(x-y)$ to that of $\lambda$ and $r$, we have

$$
\begin{aligned}
& \mathbb{E}\left[I_{n}-I_{m}\right]^{2} \\
& =\int_{0}^{T} \iint_{\mathbb{R}^{d}} \mathbb{E}\left[\lambda\left(s, y+\frac{x}{n}\right) \lambda\left(s, y+\frac{z}{n}\right)\right. \\
& \times r\left(y+\frac{x}{n}, y+\frac{z}{n}\right)-\lambda\left(s, y+\frac{x}{n}\right) \\
& \left.\times \lambda\left(s, y+\frac{z}{m}\right) r\left(y+\frac{x}{n}, y+\frac{z}{m}\right)\right] \\
& \times \phi(x) \phi(z) \mathrm{d} x \mathrm{~d} z \mathrm{~d} s \\
& +\int_{0}^{T} \iint_{\mathbb{R}^{d}} \mathbb{E}\left[\lambda\left(s, y+\frac{x}{m}\right) \lambda\left(s, y+\frac{z}{m}\right)\right. \\
& \times r\left(y+\frac{x}{m}, y+\frac{z}{m}\right)-\lambda\left(s, y+\frac{x}{m}\right) \\
& \left.\times \lambda\left(s, y+\frac{z}{n}\right) r\left(y+\frac{x}{m}, y+\frac{z}{n}\right)\right] \\
& \times \phi(x) \phi(z) \mathrm{d} x \mathrm{~d} z \mathrm{~d} s \\
& =: I_{1}+I_{2} \text {. }
\end{aligned}
$$

$I_{1}$ can be written as

$$
\begin{aligned}
I_{1}=\int_{0}^{T} \iint_{\mathbb{R}^{d}} \mathbb{E}\{ & \lambda\left(s, y+\frac{x}{n}\right) \\
& \times\left(\lambda\left(s, y+\frac{z}{n}\right)\right. \\
& \times\left[r\left(y+\frac{x}{n}, y+\frac{z}{n}\right)\right. \\
& \left.-r\left(y+\frac{x}{n}, y+\frac{z}{m}\right)\right] \\
+ & r\left(y+\frac{x}{n}, y+\frac{z}{m}\right) \\
\times & {\left[\lambda\left(s, y+\frac{z}{n}\right)\right.} \\
& \left.\left.\left.-\lambda\left(s, y+\frac{z}{m}\right)\right]\right)\right\}
\end{aligned}
$$

$\times \phi(x) \phi(z) \mathrm{d} x \mathrm{~d} z \mathrm{~d} s$.

Now, we let $r(x, y)$ be a continuous function of $x$ and $y$. We also assume that there is a $\varepsilon_{0}>0$ such that

$$
\begin{gathered}
\sup _{|y-x| \leq \varepsilon_{0}} \mathbb{E} \int_{0}^{T}\left[\lambda(s, y)^{2}\right] \mathrm{d} s<\infty, \\
\lim _{\varepsilon \rightarrow 0} \sup _{|y-x| \leq \varepsilon} \mathbb{E} \int_{0}^{T}\left[[\lambda(s, y)-\lambda(s, x)]^{2}\right] \mathrm{d} s=0 .
\end{gathered}
$$

We write $I_{1}=I_{11}+I_{12}$, where $I_{11}$ and $I_{12}$ are defined and estimated as follows. For $I_{11}$, we have

$$
\begin{aligned}
& I_{11}:=\int_{0}^{T} \iint_{\mathbb{R}^{d}} \mathbb{E}\left\{\lambda\left(s, y+\frac{x}{n}\right) \lambda\left(s, y+\frac{z}{n}\right)\right\} \\
& \times {\left[r\left(y+\frac{x}{n}, y+\frac{z}{n}\right)-r\left(y+\frac{x}{n}, y+\frac{z}{m}\right)\right] } \\
& \times \phi(x) \phi(z) \mathrm{d} x \mathrm{~d} z \mathrm{~d} s \\
& \leq C \sup _{|x| \leq 1,|z| \leq 1}\left|r\left(y+\frac{x}{n}, y+\frac{z}{n}\right)-r\left(y+\frac{x}{n}, y+\frac{z}{m}\right)\right| \\
& \times \int_{0}^{T} \iint_{\mathbb{R}^{d}} \mathbb{E}\left[\lambda\left(s, y+\frac{x}{n}\right)\right]^{2} \phi(x) \phi(z) \mathrm{d} s \mathrm{~d} x \mathrm{~d} z \\
& \leq C \sup _{|x| \leq 1,|z| \leq 1}\left|r\left(y+\frac{x}{n}, y+\frac{z}{n}\right)-r\left(y+\frac{x}{n}, y+\frac{z}{m}\right)\right| \\
& \quad \times \int_{\mathbb{R}^{d}} \int_{0}^{T} \mathbb{E}\left[\lambda\left(s, y+\frac{x}{n}\right)\right]^{2} \mathrm{~d} s \phi(x) \mathrm{d} x \\
& \leq C \quad \sup _{|x| \leq 1,|z| \leq 1}\left|r\left(y+\frac{x}{n}, y+\frac{z}{n}\right)-r\left(y+\frac{x}{n}, y+\frac{z}{m}\right)\right| \\
& \quad \times \sup _{|x| \leq 1} \int_{0}^{T} \mathbb{E}\left[\lambda\left(s, y+\frac{x}{n}\right)\right]^{2} \mathrm{~d} s
\end{aligned}
$$

$\longrightarrow 0$. 
For $I_{12}$, we have $\left(\right.$ recall $\left.K=[-1,1]^{d}\right)$

$$
\begin{aligned}
& I_{12}:=\int_{0}^{T} \iint_{\mathbb{R}^{d}} \mathbb{E}\left\{\lambda\left(s, y+\frac{x}{n}\right)\right. \\
& \times r\left(y+\frac{x}{n}, y+\frac{z}{m}\right) \\
& \times\left[\lambda\left(s, y+\frac{z}{n}\right)\right. \\
& \left.\left.-\lambda\left(s, y+\frac{z}{m}\right)\right]\right\} \\
& \times \phi(x) \phi(z) \mathrm{d} x \mathrm{~d} z \mathrm{~d} s \\
& \leq C \int_{0}^{T} \iint_{K} \mathbb{E}\left\{\lambda\left(s, y+\frac{x}{n}\right)\right. \\
& \times\left[\lambda\left(s, y+\frac{z}{n}\right)\right. \\
& \left.\left.-\lambda\left(s, y+\frac{z}{m}\right)\right]\right\} \mathrm{d} x \mathrm{~d} z \mathrm{~d} s \\
& \leq C\left\{\int_{0}^{T} \int_{K} \mathbb{E} \lambda^{2}\left(s, y+\frac{x}{n}\right) \mathrm{d} x \mathrm{~d} s\right\}^{1 / 2} \\
& \times\left\{\int _ { 0 } ^ { T } \int _ { K } \mathbb { E } \left[\lambda\left(s, y+\frac{z}{n}\right)\right.\right. \\
& \left.\left.-\lambda\left(s, y+\frac{z}{m}\right)\right]^{2} \mathrm{~d} z \mathrm{~d} s\right\}^{1 / 2} \\
& \leq C\left\{\sup _{|x| \leq 1} \int_{0}^{T} \mathbb{E} \lambda^{2}\left(s, y+\frac{x}{n}\right) \mathrm{d} x\right\}^{1 / 2} \mathrm{~d} s \\
& \times\left\{\operatorname { s u p } _ { | x | \leq 1 } \int _ { 0 } ^ { T } \mathbb { E } \left[\lambda\left(s, y+\frac{z}{n}\right)\right.\right. \\
& \left.\left.-\lambda\left(s, y+\frac{z}{m}\right)\right]^{2} \mathrm{~d} s\right\}^{1 / 2}
\end{aligned}
$$

$\longrightarrow 0$.

Thus, we have $I_{1}$ converging to 0 .

In the same way, we can show that $I_{2}$ converges to 0 under the same conditions.

Theorem 1. Let $\dot{B}_{k}(t, x)$ be a Gaussian noise which is white in time and correlated in space with covariance $r_{k}(x, y)$. Assume that $r(x, y)$ is a continuous function of $x$ and $y$. Let $(\lambda(t, x), 0 \leq$ $t \leq T, x \in \mathbb{R}^{d}$ ) be an $\mathscr{F}_{t}$-adapted processes such that conditions (14) hold. Then, the stochastic integral $\int_{0}^{T} \int_{\mathbb{R}^{d}} \lambda(t, x) \delta(x-$ $y) B_{k}(d s, d x)$ exists and

$$
\begin{gathered}
\mathbb{E}\left[\int_{0}^{T} \int_{\mathbb{R}^{d}} \lambda(s, x) \delta(x-y) B_{k}(d s, d x)\right]^{2} \\
\quad=r_{k}(y, y) \int_{0}^{T} \mathbb{E}[\lambda(s, y)]^{2} d s .
\end{gathered}
$$

Proof. The above argument shows the existence of the stochastic integral $\int_{0}^{T} \int_{\mathbb{R}^{d}} \lambda(t, x) \delta(x-y) B_{k}(\mathrm{~d} s, \mathrm{~d} x)$. We have

$$
\begin{gathered}
\mathbb{E}\left[I_{n}\right]^{2}=\int_{0}^{T} \iint_{\mathbb{R}^{d}} \mathbb{E}[\lambda(s, x) \lambda(s, z)] \\
\times \phi_{n}(x-y) \phi_{n}(z-y) \\
\quad \times r_{k}(x, z) \mathrm{d} x \mathrm{~d} z \mathrm{~d} s \\
=\int_{0}^{T} \iint_{\mathbb{R}^{d}} \mathbb{E}\left[\lambda\left(s, y+\frac{x}{n}\right) \lambda\left(s, y+\frac{z}{n}\right)\right. \\
\left.\quad \times r_{k}\left(y+\frac{x}{n}, y+\frac{z}{n}\right)\right] \\
\times \phi(x) \phi(z) \mathrm{d} x \mathrm{~d} z \mathrm{~d} s .
\end{gathered}
$$

Now, the Fatou lemma yields (17).

We also need to bound general moments of the stochastic integral $\int_{0}^{T} \int_{\mathbb{R}^{d}} \lambda(t, x) \delta(x-y) B_{k}(\mathrm{~d} s, \mathrm{~d} x)$. We have the following Burkholder type inequality.

Theorem 2. Let $\dot{B}_{k}(t, x)$ be a Gaussian noise which is white in time and correlated in space with covariance $r_{k}(x, y)$. Assume that $r(x, y)$ is a continuous function of $x$ and $y$. Let $(\lambda(t, x), 0 \leq$ $t \leq T, x \in \mathbb{R}^{d}$ ) be an $\mathscr{F}_{t}$-adapted processes such that

$$
\begin{gathered}
\sup _{|y-x| \leq \varepsilon_{0}} \mathbb{E}\left(\int_{0}^{T}\left[\lambda(s, y)^{2}\right] d s\right)^{p / 2}<\infty, \\
\lim _{\varepsilon \rightarrow 0} \sup _{|y-x| \leq \varepsilon} \mathbb{E}\left(\int_{0}^{T}\left[[\lambda(s, y)-\lambda(s, x)]^{2}\right] d s\right)^{p / 2}=0 .
\end{gathered}
$$

Then, the stochastic integral $\int_{0}^{T} \int_{\mathbb{R}^{d}} \lambda(t, x) \delta(x-y) B_{k}(d s, d x)$ exists and

$$
\begin{aligned}
& \mathbb{E}\left[\int_{0}^{T} \int_{\mathbb{R}^{d}} \lambda(s, x) \delta(x-y) B_{k}(d s, d x)\right]^{p} \\
& \leq C_{p} r_{k}^{p / 2}(y, y) \mathbb{E}\left(\int_{0}^{T}[\lambda(s, y)]^{2} d s\right)^{p / 2} .
\end{aligned}
$$

Now, we turn to consider the existence and uniqueness of the solution to the stochastic wave equation (2). We will follow the idea of mild solution. Since the Green function is more sophisticated to study in the dimension higher than 1, we will only study the one space dimensional wave equation in this paper. Higher dimension case needs much more care. Consider

$$
\begin{aligned}
\frac{\partial^{2} u(t, x)}{\partial t^{2}}-\frac{\partial^{2} u(t, x)}{\partial x^{2}}= & b(t, x, u(t, x)) \\
& +\sigma_{0}(t, x, u(t, x)) \dot{B}_{0}(t, x) \\
& +\sum_{k=1}^{N} \sigma_{k}(t, x, u(t, x)) \\
& \times \delta\left(x-y_{k}\right) \dot{B}_{k}(t, x) \\
u(0, x)=v_{0}(x), \quad & \frac{\partial u}{\partial t}(0, x)=\bar{v}_{0}(x)
\end{aligned}
$$


In the one-dimensional case, the associated fundamental solution (Green's function) of the wave operator $\partial^{2} / \partial t^{2}-$ $\partial^{2} / \partial x$ is

$$
G(t, x, y):=\frac{1}{2} I_{\{|x-y| \leq t\}} .
$$

First, we give the following definition about the solution.

Definition 3. A random field $\{u(t, x), t>0, x \in \mathbb{R}\}$ is called a solution to the stochastic wave equation if it satisfies the following identity:

$$
\begin{aligned}
u(t, x)= & \sum_{k=1}^{N} \int_{0}^{t} \int_{\mathbb{R}} G(t-s, x-z) \\
& \quad \times \sigma_{k}(s, z, u(s, z)) \delta\left(z-y_{k}\right) B_{k}(\mathrm{~d} s, \mathrm{~d} z) \\
& +\int_{0}^{t} \int_{\mathbb{R}} G(t-s, x-z) b(s, z, u(s, z)) \mathrm{d} s \mathrm{~d} z \\
& +\frac{\mathrm{d}}{\mathrm{d} t} \int_{\mathbb{R}} G(t, x-z) v_{0}(z) \mathrm{d} z \\
& +\int_{\mathbb{R}} G(t, x-y) \bar{v}_{0}(z) \mathrm{d} z
\end{aligned}
$$

Theorem 4. Assume that $b(t, x, u)$ and $\sigma_{k}(t, x, u), k=$ $0,1, \ldots, N$, satisfy the global Lipschitz condition and the linear growth condition in $u$ uniformly in $t \in[0, T]$ and $x \in \mathbb{R}$. Assume that $v_{0}$ is bounded continuous functions in $\mathbb{R}$ and $\bar{v}_{0}$ is bounded continuously differentiable functions in $\mathbb{R}$ with bounded derivative. Then, there is a unique solution to (21).

Proof. Since the solution of wave equation has the past-light cone property (see [6], p. 63), we can study the solution on a bounded domain. To simplify the presentation, we assume $b=0$.

Let us define $\mathbb{B}_{p}$ as the set of all mappings $u:[0, T] \times \mathbb{R} \times$ $\Omega \rightarrow \mathbb{R}$ such that $u(t, x, \omega)$ is continuous in $(t, x) \in[0, T] \times \mathbb{R}$ for almost all $\omega \in \Omega$ and

$$
\sup _{0 \leq t \leq T, x \in \mathbb{R}} \mathbb{E}|u(t, x)|^{p}<\infty .
$$

It is clear that $\mathbb{B}_{p}$ is a Banach space with the norm

$$
\|u\|_{\mathbb{B}_{p}}:=\sup _{0 \leq t \leq T, x \in \mathbb{R}}\left(\mathbb{E}|u(t, x)|^{p}\right)^{1 / p} .
$$

We will prove the existence and uniqueness of the solutions in $\mathbb{B}=\cap_{p \geq 2} \mathbb{B}_{p}$ to (21) by the Picard iteration. We define $\left\{u_{i}\right\}_{i=0}^{\infty}$ recursively by the following:

$$
\begin{array}{rl}
u_{i}(t, x)=T\left(u_{i-1}\right)( & t, x) \\
:=\sum_{k=1}^{N} \int_{0}^{t} \int_{\mathbb{R}} & G(t-s, x-z) \\
& \times \sigma_{k}\left(s, z, u_{i-1}(s, z)\right) \\
& \times \delta\left(z-y_{k}\right) B_{k}(\mathrm{~d} s, \mathrm{~d} z)
\end{array}
$$

$$
\begin{aligned}
& +\frac{\mathrm{d}}{\mathrm{d} t} \int_{\mathbb{R}} G(t, x-z) v_{0}(z) \mathrm{d} z \\
& +\int_{\mathbb{R}} G(t, x-y) \bar{v}_{0}(z) \mathrm{d} z
\end{aligned}
$$

for any $(t, x) \in[0, T] \times \mathbb{R}$. We also define $u_{0}=0$.

First, we show the well-posedness of the above stochastic integral for every $i=1,2, \ldots$.

For any $p>1$, let $u_{i-1} \in \mathbb{B}_{2 p}$. By the Burkholder inequality, we have

$$
\begin{aligned}
& \mathbb{E}\left|u_{i}(t, x+h)-u_{i}(t, x)\right|^{2 p} \\
& \leqslant C \sum_{k=1}^{N} r^{p}\left(y_{k}, y_{k}\right) \mathbb{E} \\
& \times\left\{\int _ { 0 } ^ { t } \left[G\left(t-s, x+h-y_{k}\right)\right.\right. \\
& \left.\quad-G\left(t-s, x-y_{k}\right)\right]^{2} \\
& \left.\quad \times\left|\sigma_{k}\left(s, y_{k}, u_{i-1}\left(s, y_{k}\right)\right)\right|^{2} \mathrm{~d} s\right\}^{p} \\
& =: C \sum_{k=1}^{N} I_{1 k} .
\end{aligned}
$$

By the Hölder inequality, we have

$$
\begin{aligned}
I_{1 k} \leqslant & C\left[\int_{0}^{t} \mid G\left(t-s, x+h-y_{k}\right)\right. \\
& \left.-\left.G\left(t-s, x-y_{k}\right)\right|^{q} \mathrm{~d} s\right]^{p / q} \\
& \times \int_{0}^{t}\left|G\left(t-s, x+h-y_{k}\right)-G\left(t-s, x-y_{k}\right)\right|^{p} \\
\leqslant & \quad \times \mathbb{E}\left|\sigma_{k}\left(s, y_{k}, u_{i-1}\left(s, y_{k}\right)\right)\right|^{2 p} \mathrm{~d} s \\
& {\left[G\left(t-s, x+h-y_{k}\right)\right.} \\
& \times \int_{0}^{t}\left|G\left(t-s, x+h-y_{k}\right)-G\left(t-s, x-y_{k}\right)\right|^{p} \mathrm{~d} s \\
\times & {\left[1+\sup _{s, y_{k}}\left|u_{i-1}\left(s, y_{k}\right)\right|^{2 p}\right] } \\
\leqslant & C|h|^{1+p / q},
\end{aligned}
$$

where $q=p /(p-1)$ is the conjugate number of $p$.

In the similar way, we can show $\mathbb{E}|u(t, x)-u(t+s, x)|^{2 p} \leqslant$ $C|s|^{1+p / q}$ for any $(t, x) \in[0, T] \times \mathbb{R}$. Thus by induction we have

$$
\mathbb{E}\left|u_{i}(t+s, x+h)-u_{i}(t, x)\right|^{2 p} \leqslant C\left(|s|^{p}+|h|^{p}\right),
$$


for all $i \in \mathbb{N}$ and $(t, x) \in[0, T] \times \mathbb{R}$. Let $\mathscr{C}_{\alpha}$ denote the set of all functions of $t \in[0, T]$ and $x \in \mathbb{R}$, which are Hölder continuous on both $t$ and $x$ on any compact subinterval of $\mathbb{R}$. Hence, by Kolmogorov's theorem, we have, for any (fixed) $0<\alpha<1 / 2, u_{i} \in \mathscr{C}_{\alpha}$ for every $i \in \mathbb{N}$. It is easy to check $\mathbb{E}|u(t, x)|^{2 p} \leqslant C$. Now, we verify easily

$$
\int_{0}^{t} \mathbb{E}\left[G(t-s, x-y) \sigma\left(s, y, u_{i}(s, y)\right)\right]^{2} \mathrm{~d} s \leqslant C .
$$

Moreover,

$$
\begin{aligned}
& \int_{0}^{t} \mathbb{E}\left[G(t-s, x-y) \sigma\left(s, y, u_{i}(s, y)\right)\right. \\
& \left.-G(t-s, x-z) \sigma\left(s, z\left(u_{i}(s, z)\right)\right)\right]^{2} \mathrm{~d} s \\
& \leqslant \int_{0}^{t} \mathbb{E}[\mid G(t-s, x-y) \\
& -\left.G(t-s, x-z)\right|^{2}\left|\sigma\left(s, y, u_{i}(s, y)\right)\right|^{2} \\
& +G^{2}(t-s, x-z) \\
& \quad \times\left(\sigma\left(s, y, u_{i}(s, y)\right)\right. \\
& \left.\left.\quad-\sigma\left(s, z, u_{i}(s, z)\right)\right)^{2}\right] \mathrm{d} s \leqslant C|y-z|
\end{aligned}
$$

for any $x \in \mathbb{R}$. Thus, $G(t-s, x-y) \sigma\left(s, y, u_{i}(s, x)\right), i=$ $1,2, \ldots, N$, satisfy conditions (14). Therefore, (26) is welldefined for all $i \in \mathbb{N}$.

Next, we show that $\left\{u_{i}, i=1,2, \ldots\right\}$ is a Cauchy sequence in $\mathbb{B}_{p}$ for any $p$. For $n, m \in \mathbb{N}$, by Burkholder's inequality and Jensen's inequality, we have

$$
\begin{aligned}
& \mathbb{E}\left|u_{n}(t, x)-u_{m}(t, x)\right|^{2 p} \\
& \leqslant C \sum_{k=1}^{N} r^{p}\left(y_{k}, y_{k}\right) \mathbb{E}\left\{\int_{0}^{t} G^{2}\left(t-s, x-y_{k}\right)\right. \\
& \quad \times\left(\sigma_{k}\left(s, y_{k}, u_{n-1}\left(s, y_{k}\right)\right)\right. \\
& \left.\left.\quad-\sigma_{k}\left(s, y_{k}, u_{m-1}\left(s, y_{k}\right)\right)\right)^{2} \mathrm{~d} s\right\}^{p} \\
& \leqslant C \sum_{k=1}^{N} r^{p}\left(y_{k}, y_{k}\right) \quad \\
& \quad \times \int_{0}^{t} \mathbb{E}\left|u_{n-1}\left(s, y_{k}\right)-u_{m-1}\left(s, y_{k}\right)\right|^{2 p} \mathrm{~d} s .
\end{aligned}
$$

Let us denote by $\mathbb{B}_{p, t}$ the set of all mappings $u:[0, t] \times \mathbb{R} \times$ $\Omega \rightarrow \mathbb{R}$ such that $u(s, x, \omega)$ is continuous in $(s, x) \in[0, t] \times \mathbb{R}$ for almost all $\omega \in \Omega$ and

$$
\sup _{0 \leq t \leq T, x \in \mathbb{R}} \mathbb{E}|u(t, x)|^{p}<\infty .
$$

We also denote

$$
\|u\|_{\mathbb{B}_{p, t}}:=\sup _{0 \leq s \leq t, x \in \mathbb{R}}\left(\mathbb{E}|u(s, x)|^{p}\right)^{1 / p} .
$$

Thus, (32) can be written as

$$
\left\|u_{n}-u_{m}\right\|_{\mathbb{B}_{p, t}^{2 p}}^{2 p} \leq C \int_{0}^{t}\left\|u_{n}-u_{m}\right\|_{\mathbb{B}_{p, s}^{2 p}}^{2 p} \mathrm{~d} s .
$$

Now, a routine argument shows that $u_{n}$ is a Cauchy sequence in $\mathbb{B}_{2 p}$. The limit of this sequence is denoted by $u$.

Letting $i \rightarrow \infty$ in (29), we have

$$
\mathbb{E}|u(t+s, x+h)-u(t, x)|^{2 p} \leqslant C\left(|s|^{p}+|h|^{p}\right)
$$

and $\sup _{x \in \mathbb{R}} \mathbb{E}|u(t, x)|^{2 p} \leqslant C$. This implies that the stochastic integral $\int_{0}^{t} G(t-s, x-y) \sigma_{k}(s, y,(u(s, y))) \delta\left(y-y_{k}\right) B_{k}(\mathrm{~d} s, \mathrm{~d} y)$ is well-defined. It is easy to see

$$
\begin{aligned}
\sup _{0 \leq t \leq T, x \in \mathbb{R}} \mathbb{E} \mid \int_{0}^{t} G(t-s, x-y) \delta\left(y-y_{k}\right) \\
\times\left(\sigma_{k}\left(s, y,\left(u_{i}(s, y)\right)\right)-\sigma_{k}(s, y,(u(s, y)))\right) \\
\times\left. B_{k}(\mathrm{~d} s, \mathrm{~d} y)\right|^{2 p} \longrightarrow 0
\end{aligned}
$$

as $i \rightarrow \infty$. Now, letting $i$ tend to infinity on both sides of (26), we see that $u$ satisfies (23). The uniqueness can be proved in similar way. Thus, we complete the proof of this theorem.

\section{Some Preliminaries for Estimation of the Point Sources}

To simplify the presentation of estimation method, we assume $\sigma_{0}(t, x, u)=0$ in (21). We also assume that the noises are space independent. Without loss of generality, we also assume $v_{0}(x)=\bar{v}_{0}(x)=0$. Moreover, we assume that $\sigma_{k}(t, x, u)=\rho_{k}$. This means we will consider the following stochastic wave equation:

$$
\begin{gathered}
\frac{\partial^{2} u(t, x)}{\partial t^{2}}-\frac{\partial^{2} u(t, x)}{\partial x^{2}}=\sum_{k=1}^{N} \rho_{k} \delta\left(x-y_{k}\right) \dot{B}_{k}(t) \\
u(0, x)=0, \quad \frac{\partial u}{\partial t}(0, x)=0 .
\end{gathered}
$$

From the Duhamel principle, we know that the solution of one-dimensional stochastic wave equations (38) is given by

$$
\begin{aligned}
u(t, x) & =\int_{0}^{t} \int_{R} 1_{\{|x-y| \leq t-s\}}(y, s) \sum_{k=1}^{N} \rho_{k} \delta\left(y-y_{k}\right) \mathrm{d} y \mathrm{~d} B_{s}^{k} \\
& =\sum_{k=1}^{N} \int_{0}^{t} \int_{R} \rho_{k} 1_{\{|x-y| \leq t-s\}}(y, s) \delta\left(y-y_{k}\right) \mathrm{d} y \mathrm{~d} B_{s}^{i} .
\end{aligned}
$$

In the remainder of this paper, we assume that the parameters $N, y_{1}, \ldots, y_{N} \rho_{1}, \ldots, \rho_{N}$ are unknown. However, at some fixed location $x \in \mathbb{R}$, we can observe the sound wave signal $\{u(t, x), 0<t \leqslant T\}$ continuously over the time interval 
$[0, T]$. We would like to use $\{u(t, x), 0<t \leqslant T\}$ to identify $N$, $y_{k}$, and $\rho_{k}, k=1,2, \ldots, N$. If one can observe the sound wave signals at some other locations $x_{1}, \ldots, x_{m}$, we can use the similar approach to (better) estimate $N, y_{1}, \ldots, y_{N} \rho_{1}, \ldots, \rho_{N}$ from all the observations $\left\{u\left(t, x_{1}\right), \ldots, u\left(t, x_{m}\right), 0<t \leqslant T\right\}$.

Put $\alpha_{k}=\left|y_{k}-x\right|, k=1,2, \ldots, N$. We arrange the real positive numbers $\alpha_{k}$ in increasing order. For example, we can assume $\alpha_{1}<\alpha_{2}<\cdots<\alpha_{N}$.

If $t<\alpha_{1}$, then, for any $k$ and $s \leq t, \alpha_{1}=\left|y_{k}-x\right| \leq t-s$ cannot hold, and hence $u(t, x)=0$.

If $\alpha_{1} \leq t<\alpha_{2}$ and $0<s<t-\left|y_{1}-x\right|=t-\alpha_{1}$, then $y_{1} \in\{y,|y-x| \leq t-s\}$; hence

$$
u(t, x)=\int_{0}^{t-\alpha_{1}} \rho_{1} \mathrm{~d} B_{s}^{1}=\rho_{1} B_{t-\alpha_{1}}^{1} .
$$

Similarly, we have, for $\alpha_{2} \leq t<\alpha_{3}$,

$$
u(t, x)=\rho_{1} B_{t-\alpha_{1}}^{1}+\rho_{2} B_{t-\alpha_{2}}^{2} ;
$$

for $\alpha_{N-1} \leq t<\alpha_{N}$

$$
u(t, x)=\rho_{1} B_{t-\alpha_{1}}^{1}+\rho_{2} B_{t-\alpha_{2}}^{2}+\cdots+\rho_{N-1} B_{t-\alpha_{N-1}}^{N-1} ;
$$

and, for $\alpha_{N} \leq t$,

$$
u(t, x)=\rho_{1} B_{t-\alpha_{1}}^{1}+\rho_{2} B_{t-\alpha_{2}}^{2}+\cdots+\rho_{N} B_{t-\alpha_{N}}^{N} .
$$

Now, for any process $Z_{t}$, we define its quadratic variation process (if it exists) as

$$
[Z, Z]_{t}=[Z]_{t}=\lim _{|\pi| \rightarrow 0} \sum_{j=0}^{n-1}\left(Z_{t_{j+1}}-Z_{t_{j}}\right)^{2}
$$

where $\pi: 0=t_{0}<t_{1}<\cdots<t_{n}=t$ is a partition of the interval $[0, t]$ and $|\pi|=\max _{0 \leq j \leq n-1}\left(t_{j+1}-t_{j}\right)$. For $d$ dimensional Brownian motion $B=\left\{B_{1}, \ldots, B_{d}\right\}$, we know that $\left[B_{i}, B_{j}\right]_{t}=t \delta_{i j}$ for $i, j=1,2, \ldots, d$ and any $t>0$, where $\delta_{i j}=1$, if $i=j, 0$, otherwise.

The quadratic variation process of $u(t, x)$ is given by

$$
h(t) \triangleq[u(\cdot, x)]_{t}=\left\{\begin{array}{cc}
0, & t<\alpha_{1} \\
\rho_{1}\left(t-\alpha_{1}\right), & \alpha_{1} \leq t<\alpha_{2} ; \\
\vdots & \vdots \\
\sum_{k=1}^{N-1} \rho_{k}\left(t-\alpha_{k}\right) & \alpha_{N-1} \leq t<\alpha_{N} ; \\
\sum_{k=1}^{N} \rho_{k}\left(t-\alpha_{k}\right), & \alpha_{N} \leq t .
\end{array}\right.
$$

Since we can observe $\{u(t, x)\}$ at the space location $x$ continuously from the time interval $[0, T]$, we know that the quadratic variation process $h(t), 0 \leq t \leq T$ is also an observable.
Let $T$ be large enough; for example, $T>\alpha_{N}$. We denote $\alpha_{0}=0, \alpha_{N+1}=T$. On $[0, T], h(t)$ has its second-order distributional derivative by

$$
h^{\prime \prime}(t)=\sum_{k=1}^{N} \rho_{k} \delta\left(t-\alpha_{k}\right) .
$$

For $g \in C^{2}[0, T]$ with $g(0)=0$, we can define linear operator $\mathscr{R}(g)$, which is called the reciprocity gap functional as follows:

$$
\mathscr{R}(g) \triangleq \int_{0}^{T} h(t) g^{\prime \prime}(t) \mathrm{d} t-h(T) g^{\prime}(T)+h^{\prime}(T) g(T) .
$$

By the integration by parts formula, we have

$$
\mathscr{R}(g)=\sum_{k=1}^{N} \rho_{k} g\left(\alpha_{k}\right) .
$$

For any function independent of the unknown parameters, we know from the definition that $\mathscr{R}(g)$ is also independent of the unknown parameters. Namely, $\mathscr{R}(g)$ is observable.

To obtain our estimators for the parameters, we take

$$
g_{n}(t)=\left(\sin \frac{\pi t}{2 T}\right)^{n}, \quad n=1,2, \ldots
$$

Then,

$$
\mathscr{R}\left(g_{n}\right)=\sum_{k=1}^{N} \rho_{k}\left(\sin \frac{\pi \alpha_{k}}{2 T}\right)^{n}=\sum_{k=1}^{N} \rho_{k} S_{k}^{n},
$$

where $S_{k}=\sin \left(\pi \alpha_{k} / 2 T\right)$.

Clearly, since $\left\{\mathscr{R}\left(g_{n}\right), n=1,2, \ldots\right\}$ are constructed from $u(t, x)$, we see that they are observable. Furthermore, once we know $S_{k}$, then we can get $\alpha_{k}$ from the identity $\alpha_{k}=$ $(2 T / \pi) \arcsin \left(S_{k}\right)$.

The $S_{k}$ can be obtained from $\mathscr{R}\left(g_{n}\right)$ by the following way. We let $H_{\mu}(\mu=0,1)$ be the $N \times N$ matrices of the following forms:

$$
H_{\mu}=\left(\begin{array}{cccc}
\mathscr{R}\left(g_{\mu}\right) & \mathscr{R}\left(g_{\mu+1}\right) & \cdots & \mathscr{R}\left(g_{\mu+N-1}\right) \\
\mathscr{R}\left(g_{\mu+1}\right) & \mathscr{R}\left(g_{\mu+2}\right) & \cdots & \mathscr{R}\left(g_{\mu+N}\right) \\
\vdots & \vdots & \vdots & \vdots \\
\mathscr{R}\left(g_{\mu+N-1}\right) & \mathscr{R}\left(g_{\mu+N}\right) & \cdots & \mathscr{R}\left(g_{\mu+2 N-2}\right)
\end{array}\right) .
$$

The following result is from [7, 8]. For more details, see $[7,8]$ and references therein.

Theorem 5. Let $H_{\mu}(\mu=0,1)$ be defined above; then $S_{1}, S_{2}, \ldots, S_{N}$ are the eigenvalues of Hermite matrix $H_{0}^{-1} H_{1}$.

Proof. First, we will introduce some intermediate quantities as follows. For $j \in N$, we define

$$
A_{j}=\left(\begin{array}{cccc}
\left(S_{1}\right)^{j} & \left(S_{2}\right)^{j} & \cdots & \left(S_{N}\right)^{j} \\
\left(S_{1}\right)^{j+1} & \left(S_{2}\right)^{j+1} & \cdots & \left(S_{N}\right)^{j+1} \\
\vdots & \vdots & \cdots & \vdots \\
\left(S_{1}\right)^{N+j-1} & \left(S_{2}\right)^{N+j-1} & \cdots & \left(S_{N}\right)^{N+j-1}
\end{array}\right)_{N \times N} .
$$


We denote the diagonal matrix

$$
D=\operatorname{diag}\left(S_{1}, S_{2}, \ldots, S_{N}\right) .
$$

We define the vectors

$$
\mu_{j}=\left(\begin{array}{c}
\mathscr{R}\left(g_{j}\right) \\
\mathscr{R}\left(g_{j+1}\right) \\
\vdots \\
\mathscr{R}\left(g_{N+j-1}\right)
\end{array}\right)_{N \times 1}
$$

and vector $\rho=\left(\rho_{1}, \rho_{2}, \ldots, \rho_{N}\right)^{T}$.

It is obvious that, for all $j \geqslant 0$, we have

$$
\mu_{j}=A_{j} \rho, \quad A_{j+1}=A_{j} D .
$$

Thus, for all $j$, we have

$$
\mu_{j+1}=A_{0} D^{j+1} \rho=A_{0} D A_{0}^{-1} A_{0} D^{j} \rho=A_{0} D A_{0}^{-1} \mu_{j} .
$$

Denote $\Gamma=A_{0} D A_{0}^{-1}$. Then, $\mu_{j+1}=\Gamma \mu_{j}$ and the matrix $\Gamma$ has the $S_{k}$ as its eigenvalue corresponding to the eigenvector $\beta_{k}=\left(1, S_{k}^{1}, \ldots, S_{k}^{N-1}\right)^{T}$ for $k=1,2, \ldots, N$.

On the other hand, we have

$$
H_{1}=\left(\mu_{1}, \mu_{2}, \ldots, \mu_{N}\right)=\Gamma\left(\mu_{0}, \mu_{1}, \ldots, \mu_{N-1}\right)=\Gamma H_{0} .
$$

Because $A_{0}$ is a Vandermonde matrix and $S_{1}, S_{2}, \ldots, S_{N}$ are assumed to be distinct, we also can show that vectors $\mu_{0}, \mu_{1}, \ldots, \mu_{N-1}$ are independent, which means that $H_{0}$ is invertible. Hence, $\Gamma=H_{1} H_{0}^{-1}$. The conclusion follows.

Remark 6. Let $M>N$ be integers. Defining $\mu_{j}(M)=\left(\mathscr{R}\left(g_{j}\right)\right.$, $\left.\mathscr{R}\left(g_{j+1}\right), \ldots, \mathscr{R}\left(g_{M+j-1}\right)\right)^{T}$ (T denotes the transpose), one can see that the vectors $\mu_{0}(M), \mu_{1}(M), \ldots, \mu_{M-1}(M)$ are images of $\rho, D \rho, \ldots, D^{M-1} \rho \in R^{N}$ by the action of the matrix

$$
A_{0}(M)=\left(\begin{array}{cccc}
1 & 1 & \cdots & 1 \\
\left(S_{1}\right)^{1} & \left(S_{2}\right)^{1} & \cdots & \left(S_{N}\right)^{1} \\
\vdots & \vdots & \cdots & \vdots \\
\left(S_{1}\right)^{M-1} & \left(S_{2}\right)^{M-1} & \cdots & \left(S_{N}\right)^{M-1}
\end{array}\right)_{M \times N}
$$

from which and from the independence of the vectors $\mu_{0}(M), \mu_{1}(M), \ldots, \mu_{M-1}(M)$ one can deduce that the determinant of matrix $\left(\mu_{0}, \mu_{1}, \ldots, \mu_{M-1}\right)$ is equal to zero. For more details, see [7].

Remark 7. One can find, from the proof, that vector $\rho$ is the solution of linear equations $\mu_{0}=A_{0} \rho$.

\section{Estimations for Point Sources from Discrete Time Observations}

By Theorem 5, we know that the parameters we want to estimate are contained in the eigenvalues of Hermite matrix $H_{0}^{-1} H_{1}$.

We assume in this section that the wave signals are observed at the location $x \in \mathbb{R}$ but at discrete time instants
$0=t_{0}<t_{1}<\cdots<t_{m}=T$. We denote $\Delta=t_{j+1}-t_{j}(j=$ $0,1, \ldots, m-1)$.

We define the approximation $h_{\Delta}$ of the quadratic variations $h$ of solution process $u(t, x)$ for any $\Delta>0$. Assume that there are integers $l_{1}, l_{2}, \ldots, l_{N}$ such that $t_{l_{j}} \leqslant \alpha_{j}<t_{l_{j}+1}$ for all $j=1,2, \ldots, N$. For any $0<t<T$, since there is an integer $j_{t}$ such that $t_{j_{t}} \leqslant t<t_{j_{t}+1}$, one defines

$$
h_{\Delta}(t) \triangleq \sum_{k=1}^{j_{t}}\left|u\left(t_{k}, x\right)-u\left(t_{k-1}, x\right)\right|^{2}
$$

This is also an approximation of the quadratic variations process $h(t)$ of solution process $u(t, x)$ based on the observation time instants $0=t_{0}<t_{1}<\cdots<t_{m}=T$. We know that for $t$ fixed $h_{\Delta}(t) \stackrel{L^{2}}{\rightarrow} j t-\alpha_{1}-\alpha_{2}-\cdots-\alpha_{j}$ as $\Delta \rightarrow 0$ with $\alpha_{j} \leqslant t<\alpha_{j+1}$. In fact, we can show that convergence holds in the almost sure sense (see Lemma 10).

To use $h_{\Delta}(t)$ to obtain estimators of the parameters, we compute the following. For any twice differentiable function $g$, by Abel sum formula, we have

$$
\begin{aligned}
\int_{0}^{T} h_{\Delta}(t) g^{\prime \prime}(t) \mathrm{d} t= & h_{\Delta}(T) g^{\prime}(T) \\
& -\int_{0}^{T} g^{\prime}(t) \mathrm{d} h_{\Delta}(t)=h_{\Delta}(T) g^{\prime}(T) \\
& -\sum_{i=1}^{m} g^{\prime}\left(t_{i}\right)\left|u\left(t_{i}, x\right)-u\left(t_{i-1}, x\right)\right|^{2} \\
= & h_{\Delta}(T) g^{\prime}(T)-\left|u(T, x)-u\left(t_{M_{\Delta}-1}, x\right)\right|^{2} \\
& \times \sum_{i=1}^{m} g^{\prime}\left(t_{i}\right)+\sum_{i=1}^{m-1}\left(Z_{i+1}-Z_{i}\right) \sum_{k=1}^{i} g^{\prime}\left(t_{k}\right),
\end{aligned}
$$

where $Z_{i}=\left|u\left(t_{i}, x\right)-u\left(t_{i-1}, x\right)\right|^{2}, i=1,2, \ldots, m$. Thus, we can define

$$
\begin{aligned}
\mathscr{R}_{\Delta}(g) \triangleq & \int_{0}^{T} h_{\Delta}(t) \Delta g(t) \mathrm{d} t-h_{\Delta}(T) g^{\prime}(T) \\
& +\left|u(T, x)-u\left(t_{M_{\Delta}-1}, x\right)\right|^{2} \sum_{i=1}^{m} g^{\prime}\left(t_{i}\right) .
\end{aligned}
$$

For any nonnegative integer $\mu$, we introduce

$$
\begin{aligned}
H_{\Delta, \mu, m} & \\
= & \left(\begin{array}{cccc}
\mathscr{R}_{\Delta}\left(g_{\mu}\right) & \mathscr{R}_{\Delta}\left(g_{\mu+1}\right) & \cdots & \mathscr{R}_{\Delta}\left(g_{\mu+m-1}\right) \\
\mathscr{R}_{\Delta}\left(g_{\mu+1}\right) & \mathscr{R}_{\Delta}\left(g_{\mu+2}\right) & \cdots & \mathscr{R}_{\Delta}\left(g_{\mu+m}\right) \\
\vdots & \vdots & \vdots & \vdots \\
\mathscr{R}_{\Delta}\left(g_{\mu+m-1}\right) & \mathscr{R}_{\Delta}\left(g_{\mu+m}\right) & \cdots & \mathscr{R}_{\Delta}\left(g_{\mu+2 m-2}\right)
\end{array}\right) .
\end{aligned}
$$


First, we estimate $N$. It is known that when $m>N, H_{0, m}$ will be degenerate. However, there is a difference between $H_{\Delta, 0, m}$ and $H_{0, m}$ and there may be also error from the computer computation. To make the method robust, we introduce a small number $\delta$. We propose to estimate $N$ by the following estimator:

$$
\widehat{N}=\max \left\{m,\left|\operatorname{det}\left(H_{\Delta, 0, m}\right)\right|>\delta\right\} .
$$

As in the case of continuous time observation we let $S_{\Delta, 1}^{\uparrow}, S_{\Delta, 2}^{\uparrow}, \ldots, S_{\Delta, N}^{\uparrow}$ be the $n$ eigenvalues (in increasing order) of the Hermite matrix $\Gamma_{\Delta}=H_{\Delta, 0}^{-1} H_{\Delta, 1}$. Then, we obtain the estimations of the locations of the point sources by

$$
\widehat{\alpha}_{\Delta, k}=\left|\widehat{y}_{k}-x\right|=g_{k}^{-1}\left(S_{\Delta, k}^{\uparrow}\right),
$$

for $k=1,2, \ldots, N$. The estimation of $y_{k}$ follows from (64). Note that $g_{k}, k=1,2, \ldots$, are given by (49).

Similar to $A_{0}, \mu_{0}$ in Theorem 5 , we define

$$
\begin{gathered}
A_{0}(\Delta)=\left(\begin{array}{cccc}
1 & 1 & \cdots & 1 \\
S_{\Delta, 1}^{\uparrow} & S_{\Delta, 2}^{\uparrow} & \cdots & S_{\Delta, N}^{\uparrow} \\
\vdots & \vdots & \cdots & \vdots \\
\left(S_{\Delta, 1}^{\uparrow}\right)^{N-1} & \left(S_{\Delta, 2}^{\uparrow}\right)^{N-1} & \cdots & \left(S_{\Delta, N}^{\uparrow}\right)^{N-1}
\end{array}\right)_{N \times N}, \\
\mu_{0}(\Delta)=\left(\begin{array}{c}
\mathscr{R}_{\Delta}\left(g_{0}\right) \\
\mathscr{R}_{\Delta}\left(g_{1}\right) \\
\vdots \\
\mathscr{R}_{\Delta}\left(g_{N-1}\right)
\end{array}\right)_{N \times 1} .
\end{gathered}
$$

As in Remark 7, the estimation $\widehat{\rho}=\left(\widehat{\rho}_{1}, \widehat{\rho}_{2}, \ldots, \widehat{\rho}_{N}\right)$ of the strength $\rho$ of the point sources can be obtained by solving the following linear equation:

$$
\mu_{0}(\Delta)=A_{0}(\Delta) \hat{\rho} .
$$

Now, we can summarize the estimation procedure as follows.

Step 1. Compute $h_{\Delta}(t)$ on $[0, T]$ and $\mathscr{R}_{\Delta}\left(g_{i}\right)$ according to transformation (61).

Step 2. Identify the value of $N$ as the maximum $M$ such that

$$
\operatorname{det}\left(\mu_{0}(\Delta, M), \mu_{1}(\Delta, M), \ldots, \mu_{M-1}(\Delta, M)\right) \neq 0 .
$$

Step 3. Compute the eigenvalues $S_{\Delta, k}^{\uparrow}$ of matrix $H_{\Delta, 0}^{-1} H_{\Delta, 1}$.

Step 4. Compute the intensities by formulae $\rho(\Delta)=$ $A_{0}^{-1}(\Delta) \mu_{0}(\Delta)$.

\section{Convergence of Estimations}

In this section, we will show that the estimations obtained in the previous section converge to the true values a.s. as time space $\Delta$ tends to zero.
Theorem 8. For any function $g \in C^{2}([0, T])$ with $g(0)=0$, we have $\mathscr{R}_{\Delta}(g)$ converging to $\mathscr{R}(g)$ a.s. as $\Delta \rightarrow 0$.

This will be fulfilled by the following two lemmas.

Lemma 9. For any function $g \in C([0, T]), \int_{0}^{T} h_{\Delta}(t) g(t) d t$ converges to $\int_{0}^{T} h(t) g(t) d t$ in the sense of $L^{2}$ as $\Delta \rightarrow 0$.

Proof. It is sufficient to show that, for any function $g \in$ $C([0, T]), \int_{0}^{T} h_{\Delta}(t) g(t) \mathrm{d} t$ converges to $\int_{0}^{T} h(t) g(t) \mathrm{d} t$ in the sense of $L^{2}$ as $\Delta \rightarrow 0$. By Hölder inequality, one has, for any function $g \in C([0, T])$,

$$
\begin{aligned}
& \mathbb{E}\left(\int_{0}^{T} h_{\Delta}(t) g(t) \mathrm{d} t-\int_{0}^{T} h(t) g(t) \mathrm{d} t\right)^{2} \\
& \quad \leqslant \int_{0}^{T} \mathbb{E}\left(h_{\Delta}(t)-h(t)\right)^{2} \mathrm{~d} t \times \int_{0}^{T} g^{2}(t) \mathrm{d} t \\
& \quad=\sum_{j=1}^{N} \int_{\alpha_{j}}^{\alpha_{j+1}} \mathbb{E}\left[h_{\Delta}(t)-h(t)\right]^{2} \mathrm{~d} t \times \int_{0}^{T} g^{2}(t) \mathrm{d} t \\
& \quad=\int_{0}^{T} g^{2}(t) \mathrm{d} t \sum_{j=1}^{N} I_{j},
\end{aligned}
$$

where

$$
\begin{aligned}
I_{j}= & \int_{\alpha_{j}}^{t_{l_{j+1}}} \mathbb{E}\left(h_{\Delta}(t)-h(t)\right)^{2} \mathrm{~d} t \\
& +\sum_{k=l_{j}+1}^{l_{j+1}-1} \int_{t_{k}}^{t_{k+1}} \mathbb{E}\left(h_{\Delta}(t)-h(t)\right)^{2} \mathrm{~d} t \\
& +\int_{t_{l_{j+1}}}^{\alpha_{j+1}} \mathbb{E}\left(h_{\Delta}(t)-h(t)\right)^{2} \mathrm{~d} t .
\end{aligned}
$$

For $l_{j}+1 \leqslant k \leqslant l_{j+1}-1$, we have

$$
\begin{gathered}
\int_{t_{k}}^{t_{k+1}} \mathbb{E}\left(h_{\Delta}(t)-h(t)\right)^{2} \mathrm{~d} t \\
=\int_{t_{k}}^{t_{k+1}} \mathbb{E}\left(\sum_{i=1}^{k}\left|u\left(t_{i}, x\right)-u\left(t_{i-1}, x\right)\right|^{2}\right. \\
\left.-\left(\sum_{i=1}^{j} \rho_{i}\left(t-\alpha_{i}\right)\right)\right)^{2} \mathrm{~d} t \\
=\int_{t_{k}}^{t_{k+1}} \mathbb{E}\left(\sum_{s=1}^{j-1} \sum_{i=l_{s}+1}^{l_{s+1}}\left|u\left(t_{i}, x\right)-u\left(t_{i-1}, x\right)\right|^{2}\right. \\
+\sum_{i=l_{j}+1}^{k}\left|u\left(t_{i}, x\right)-u\left(t_{i-1}, x\right)\right|^{2} \\
\left.-\left(\sum_{i=1}^{j} \rho_{i}\left(t-\alpha_{i}\right)\right)\right)^{2} \mathrm{~d} t
\end{gathered}
$$




$$
\begin{aligned}
& =\int_{t_{k}}^{t_{k+1}} \mathbb{E}\left(\sum _ { s = 1 } ^ { j - 1 } \left(\sum_{i=l_{s}+2}^{l_{s+1}} \mid\left(\rho_{1} B_{t_{i}-\alpha_{1}}^{1}+\cdots+\rho_{s} B_{t_{i}-\alpha_{s}}^{s}\right)\right.\right. \\
& -\left.\left(\rho_{1} B_{t_{i-1}-\alpha_{1}}^{1}+\cdots+\rho_{s} B_{t_{i-1}-\alpha_{s}}^{s}\right)\right|^{2} \\
& +\mid\left(\rho_{1} B_{t_{l_{s}+1}-\alpha_{1}}^{1}+\cdots+\rho_{s} B_{t_{l_{s}+1}-\alpha_{s}}^{s}\right) \\
& \left.-\left.\left(\rho_{1} B_{t_{s}-\alpha_{1}}^{1}+\cdots+\rho_{s-1} B_{t_{l s}-\alpha_{s-1}}^{s-1}\right)\right|^{2}\right) \\
& +\left(\sum_{i=l_{j}+2}^{k} \mid\left(\rho_{1} B_{t_{i}-\alpha_{1}}^{1}+\cdots+\rho_{j} B_{t_{i}-\alpha_{j}}^{j}\right)\right. \\
& -\left.\left(\rho_{1} B_{t_{i-1}-\alpha_{1}}^{1}+\cdots+\rho_{j} B_{t_{i-1}-\alpha_{j}}^{j}\right)\right|^{2} \\
& +\mid\left(\rho_{1} B_{t_{l^{+1}}-\alpha_{1}}^{1}+\cdots+\rho_{j} B_{t_{l_{j}+1}-\alpha_{j}}^{j}\right) \\
& -\left(\rho_{1} B_{t_{l_{j}}-\alpha_{1}}^{1}+\cdots\right. \\
& \left.\left.+\rho_{j-1} B_{t_{l_{j}}-\alpha_{j-1}}^{j-1}\right)\left.\right|^{2}\right) \\
& \left.-\left(\sum_{i=1}^{j} \rho_{i}\left(t-\alpha_{i}\right)\right)\right)^{2} \mathrm{~d} t
\end{aligned}
$$

Let $X$ be a standard normal random variable. Denote the constant $\mathbb{E}\left(X^{2}-1\right)^{2}$ by $A$ (actually $A=2$ ). By the independent increments property of Brownian motion and the independence between $B^{k}$ and $B^{l}$ for $k \neq l$, we have

$$
\begin{gathered}
\int_{t_{k}}^{t_{k+1}} \mathbb{E}\left(h_{\Delta}(t)-h(t)\right)^{2} \mathrm{~d} t \\
=C \int_{t_{k}}^{t_{k+1}}\left(A \sum _ { s = 1 } ^ { j - 1 } \left(\left(l_{s+1}-l_{s}-1\right) s^{2} \Delta^{2}\right.\right. \\
\left.+\left((s-1) \Delta+t_{l_{s}+1}-\alpha_{s}\right)^{2}\right) \\
+A j^{2} \Delta^{2}\left(k-l_{j}-1\right) \\
\left.+A\left((j-1) \Delta+t_{l_{j}+1}-\alpha_{j}\right)^{2}\right) \\
+\left[\left(j t-\alpha_{1}-\cdots-\alpha_{j}\right)\right. \\
-\left(j k \Delta-l_{1} \Delta-\cdots-l_{j} \Delta+t_{l_{1}+1}\right. \\
\left.\left.-\alpha_{1}+\cdots+t_{l_{j}+1}-\alpha_{j}\right)\right]^{2} \mathrm{~d} t
\end{gathered}
$$

$$
\begin{aligned}
& \leqslant C \int_{t_{k}}^{t_{k+1}}\left(\sum_{s=1}^{j-1}\left(l_{s+1}-l_{s}\right) s^{2} \Delta^{2}\right. \\
& \left.\quad+j^{2} \Delta^{2}\left(k-l_{j}\right)+9 j^{2} \Delta^{2}\right) \mathrm{d} t \\
& =C \Delta^{2}+o\left(\Delta^{2}\right),
\end{aligned}
$$

where the constant $C$ depends only on $T, A, \alpha_{j}, \rho_{j}(j=$ $1,2, \ldots, N)$ and $o(x) / x \rightarrow 0$ as $x \rightarrow 0$. In the similar way, we can get the same estimates for the first and last terms in $I_{j}$. Since the total number of the terms in $I_{j}$ is less than $\left(\left(\alpha_{j+1}-\alpha_{j}\right) / \Delta\right)+2, I_{j} \rightarrow 0$ as $\Delta \rightarrow 0$. Hence, we complete the proof of this lemma.

Lemma 10. For any function $g \in C([0, T]), \int_{0}^{T} h_{\Delta}(t) g(t) d t$ converges to $\int_{0}^{T} h(t) g(t) d t$ a.s. as $\Delta \rightarrow 0$.

Proof. By integration by parts, we know

$$
\begin{aligned}
\int_{0}^{T} h_{\Delta}(t) g(t) \mathrm{d} t= & G(T) h_{\Delta}(T) \\
& -\sum_{k=1}^{N} G\left(t_{k}\right)\left|u\left(t_{k}, x\right)-u\left(t_{k-1}, x\right)\right|^{2},
\end{aligned}
$$

where $G(t)=\int_{0}^{t} g(s) \mathrm{d} s$. Therefore, for any $\Delta>0$, the square integrable functional $\int_{0}^{T} h_{\Delta}(t) g(t) \mathrm{d} t$ of $N$ dimensional Brownian motion $B=\left(B^{1}, B^{2}, \ldots, B^{N}\right)$ belongs to the direct sum of $R$ and the second chaos.

To this end, we define the second quantization operator $\Gamma(\alpha)$ (for $0 \leq \alpha \leq 1$ ) from $L^{2}$ to $L^{2}$ by

$$
\Gamma(\alpha) F=\sum_{n=0}^{\infty} \alpha^{n} J_{n} F,
$$

where $F \in L^{2}(\Omega)$.

Setting $m=\alpha^{-2}+1 \geq 2$, then, by the hypercontractivity of OU semigroup (see $[9,10]$ ), one has

$$
\|\Gamma(\alpha) F\|_{m} \leq\|F\|_{2} .
$$

Letting $F=\int_{0}^{T} h_{\Delta}(t) g(t) \mathrm{d} t-\int_{0}^{T} h(t) g(t) \mathrm{d} t \in \operatorname{Range}\left(J_{0} \oplus J_{2}\right)$, we have

$$
\Gamma(\alpha) F=\mathbb{E} F+\alpha^{2}(F-\mathbb{E} F)=\left(1-\alpha^{2}\right) \mathbb{E} F+\alpha^{2} F .
$$

Thus, the hypercontractivity justifies the following form:

$$
\begin{aligned}
\left\|\alpha^{2} F\right\|_{m} & \leqslant\|F\|_{2}+\left\|\left(1-\alpha^{2}\right) \mathbb{E} F\right\|_{m} \\
& \leqslant\|F\|_{2}+\left\|\left(1-\alpha^{2}\right) \mathbb{E} F\right\|_{2} \leqslant C \Delta^{1 / 2} .
\end{aligned}
$$

In the last inequality, we use the facts appearing in the end of the proof of Lemma 9. Therefore,

$$
\mathbb{E}|F|^{m} \leq C(m-1)^{m} \Delta^{m / 2} .
$$


Now, we identify the $\Delta \rightarrow 0$ with $1 / n \rightarrow 0$ as $n \rightarrow$ 0 . Denote the set $\left\{|F|>n^{-1 / 8}\right\}$ by $A_{n}, n=1,2, \ldots$. Then, $P\left(A_{n}\right) \leq C / n^{m / 2}$. Choose $m$ large enough such that $\sum_{0}^{\infty} P\left(A_{n}\right)<\infty$ holds. Thus, the Borel-Cantelli lemma can be applied to show $F=\int_{0}^{T} h_{\Delta}(t) g(t) \mathrm{d} t-\int_{0}^{T} h(t) g(t) \mathrm{d} t \rightarrow 0$ a.s. as $\Delta$ tends to zero.

Proof of Theorem 8. As the proof of Lemma 10, we just need to show that the limit holds in the sense of $L^{2}$. Actually, as $\Delta \rightarrow 0$, one has

$$
\begin{aligned}
\left|u(T, x)-u\left(t_{M_{\Delta}-1}, x\right)\right|^{2} \sum_{i=1}^{M_{\Delta}} g^{\prime}\left(t_{i}\right) \\
=\left(\left|u(T, x)-u\left(t_{M_{\Delta}-1}, x\right)\right|^{2}-N \Delta\right) \\
\quad \times \sum_{i=1}^{M_{\Delta}} g^{\prime}\left(t_{i}\right)+N \Delta \sum_{i=1}^{M_{\Delta}} g^{\prime}\left(t_{i}\right) \\
\longrightarrow N \int_{0}^{T} g^{\prime}(t) \mathrm{d} t=N g(T)=h^{\prime}(T) g(T) .
\end{aligned}
$$

Hence, the result of Theorem 8 is concluded.

Since Weyl's perturbation theorem (see $[11,12])$ says that

$$
\max _{k}\left|\lambda_{k}^{\uparrow}(\Delta)-\lambda_{k}^{\uparrow}\right| \leqslant\left\|H_{\Delta, 0}^{-1} H_{\Delta, 1}-H_{0}^{-1} H_{1}\right\|,
$$

by Theorem 8 , we can get the convergence of estimations of the locations by an obvious way, which is stated as follows.

Theorem 11. For any $k=1,2, \ldots, N$, one has $S_{\Delta, k}^{\uparrow} \rightarrow S_{k}$ a.s. as $\Delta \rightarrow 0$.

Next, we will give the convergence of estimators of the intensities of the point sources.

Theorem 12. Let $\rho(\Delta)=\left(\rho_{1}(\Delta), \rho_{2}(\Delta), \ldots, \rho_{N}(\Delta)\right)$ be the solution of linear equations $\mu_{0}(\Delta)=A_{0}(\Delta) \rho(\Delta)$; that is, $\rho(\Delta)=A_{0}^{-1}(\Delta) \mu_{0}(\Delta)$. Then, one has $\rho(\Delta) \rightarrow \rho$ a.s. as $\Delta \rightarrow 0$.

Proof. Notice that $\rho$ solves the equation $\mu_{0}=A_{0} \rho$. By Theorems 8 and 11, one can get the conclusion of this theorem.

\section{Conflict of Interests}

The authors declare that there is no conflict of interests regarding the publication of this paper.

\section{Acknowledgments}

Y. Hu was partially supported by Grant no. 209206 from the Simons Foundation and a General Research Fund (GRF) of University of Kansas. G. Rang was supported by NSFC11171262 in China and by Post 70s Project of Wuhan University.

\section{References}

[1] T. Ohe, H. Inui, and K. Ohnaka, "Real-time reconstruction of time-varying point sources in a three-dimensional scalar wave equation," Inverse Problems, vol. 27, no. 11, Article ID 115011, 2011.

[2] J. B. Walsh, "An introduction to stochastic partial differential equations," in École d'été de Probabilités de Saint-Flour, XIV1984, vol. 1180 of Lecture Notes in Mathematics, pp. 265-439, Springer, 1986.

[3] D. Khoshnevisan and F. Rassoul-Agha, Eds., A Minicourse on Stochastic Partial Differential Equations, vol. 1962 of Lecture Notes in Mathematics, Springer, Berlin, Germany, 2009.

[4] R. C. Dalang and M. Sanz-Solé, "Hölder-Sobolev regularity of the solution to the stochastic wave equation in dimension three," Memoirs of the American Mathematical Society, vol. 199, no. 931, 2009.

[5] Y. Hu, J. Y. Huang, and D. Nualart, "On Hölder continuity of the solution of stochastic waveequations," preprint, 2013.

[6] R. C. Dalang, D. Khoshnevisan, Mueller, C. Nualart D, and Y. Xiao, A Minicourse on Stochastic Partial Differential Equations, vol. 1962 of Lecture Notes in Mathematics, Springer, Berlin, Germany, 2009.

[7] A. El Badia and T. Ha-Duong, "An inverse source problem in potential analysis," Inverse Problems, vol. 16, no. 3, pp. 651-663, 2000.

[8] A. El Badia and T. Ha-Duong, "Determination of point wave sources by boundary measurements," Inverse Problems, vol. 17, no. 4, pp. 1127-1139, 2001.

[9] Z.-Y. Huang and J.-A. Yan, Introduction to Infinite Dimensional Stochastic Analysis, vol. 502, Kluwer Academic, 2000.

[10] D. Nualart, The Malliavin Calculus and Related Topics, Springer, 1995.

[11] R. Bhatia, Matrix Analysis, vol. 169, Springer, New York, NY, USA, 1997.

[12] G. W. Anderson, A. Guionnet, and O. Zeitouni, An Introduction to Random Matrices, vol. 118, Cambridge University Press, 2010. 


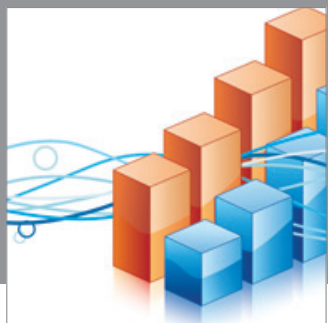

Advances in

Operations Research

mansans

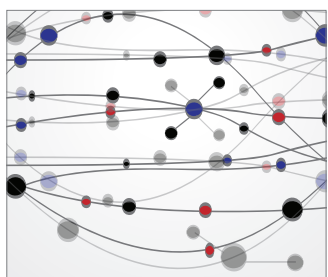

The Scientific World Journal
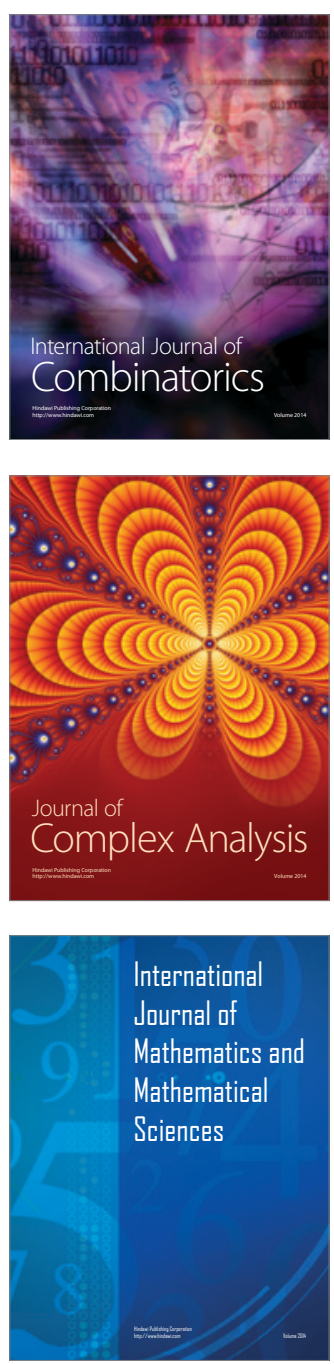
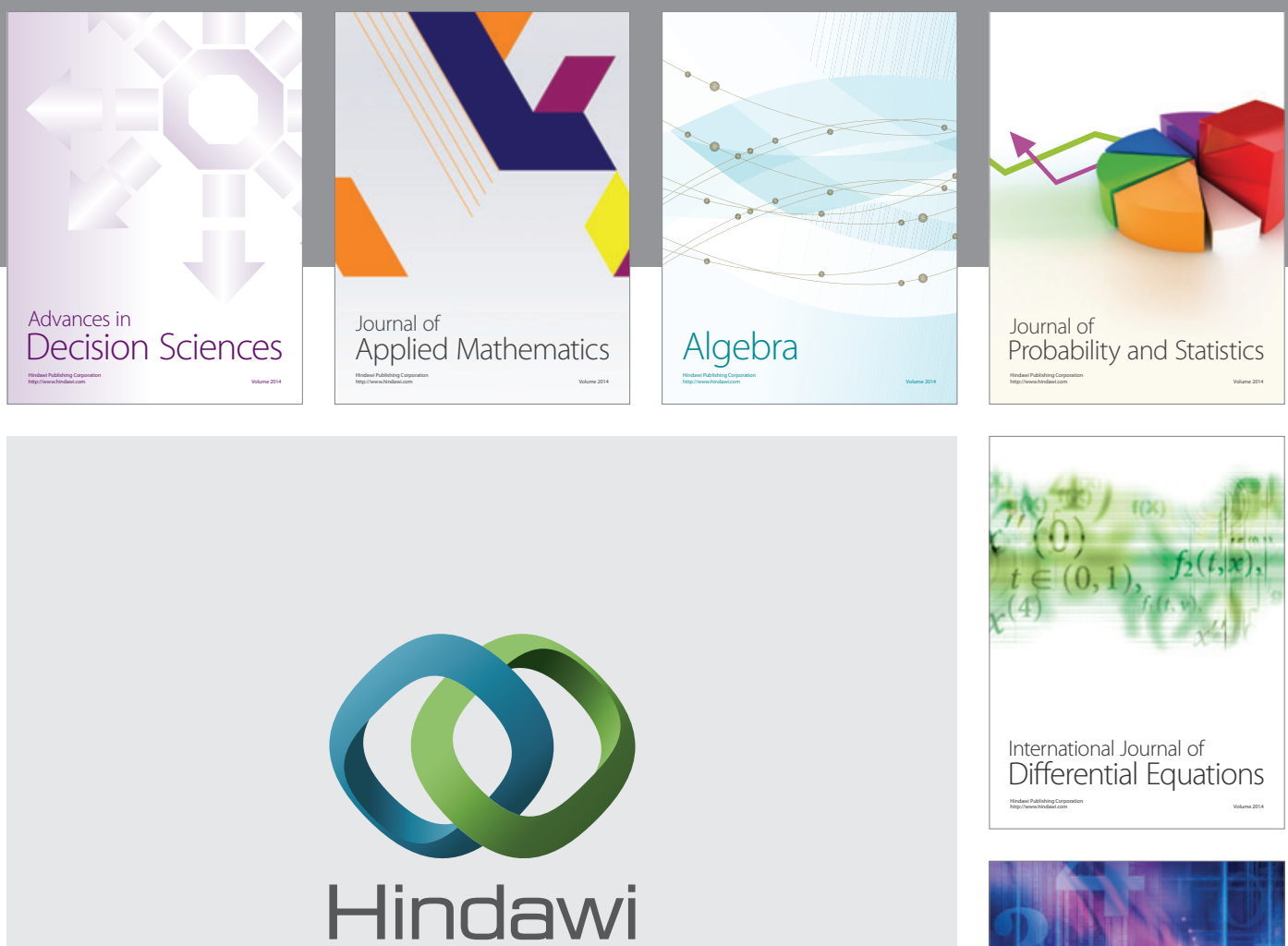

Submit your manuscripts at http://www.hindawi.com
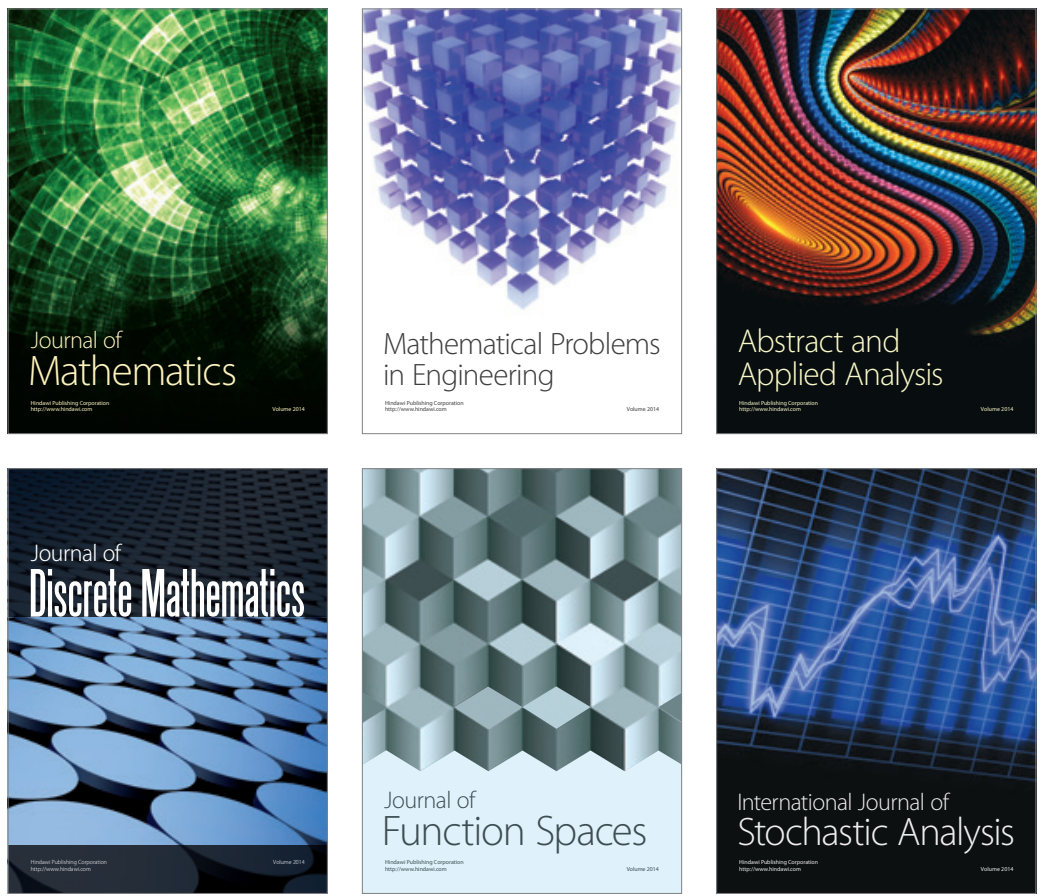

Journal of

Function Spaces

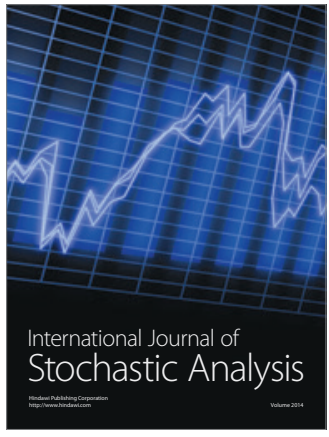

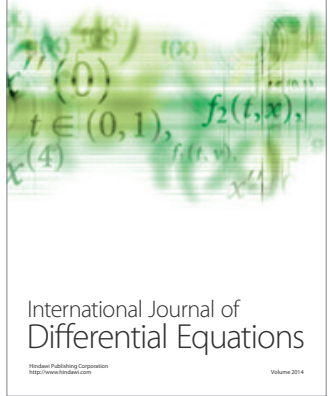
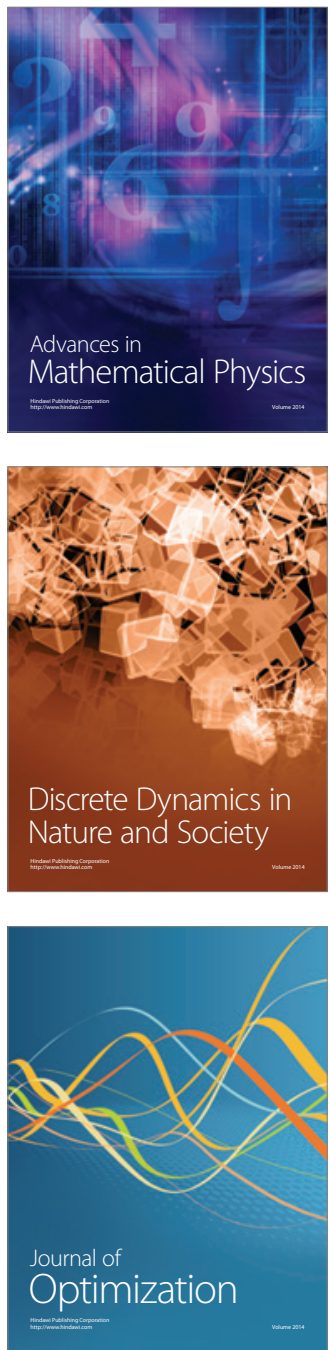